\title{
Dineutron correlations in quasi two-dimensional systems in a simplified model and possible relation to neutron-rich nuclei
}

\author{
Yoshiko Kanada-En'yo and Nobuo Hinohara \\ Yukawa Institute for Theoretical Physics, Kyoto University, Kyoto 606-8502, Japan \\ Tadahiro Suhara \\ Department of Physics, Kyoto University, Kyoto 606-8502, Japan \\ Peter Schuck \\ Institut de Physique Nucléaire, CNRS, UMR 8608, Orsay, F-91505, France \\ Université Paris-Sud, Orsay, F-91505, France and \\ Laboratoire de Physique et Modélisation des Milieux Condensés, CNR et Université Joseph Fourier, \\ 25 Av. des Martyrs, BP 166, F-38042 Grenoble Cedex 9, France
}

\begin{abstract}
Two-neutron correlation in the ${ }^{1} S$ channel in quasi two-dimensional (2D) neutron systems at zero temperature is studied by means of the BCS theory with finite-range effective nuclear forces. The dineutron correlation in low density neutron systems confined in an infinite slab is investigated in a simplified model that neutron motion of one direction is frozen. When the slab is thin enough, two neutrons form a tightly bound dineutron with a small size in the quasi-2D system, and a Bose dineutron gas is found in low density limit. With increase of Fermi momentum, the neutron system changes from the Bose-gas phase to the superfluid Cooper pair phase. The density dependence of the neutron pairing shows the BCS-BEC crossover phenomena at finite low-density region. In the transition region, the size shrinking of neutron pair and enhancement of pairing gap are found. The relation to dineutron correlation at surface of neutron-rich nuclei is also discussed.
\end{abstract}

\section{INTRODUCTION}

Two-neutron correlations in neutron-rich nuclei is presently one of the fore-front subjects in the physics of unstable nuclei. In two-neutron halo nuclei [1] such as ${ }^{11} \mathrm{Li}$, the dineutron correlation was theoretically predicted in many studies [2, 3, 4, 5, 6, 7, 8, 9, 10, 11] and is supported by experiments 12, 13, 14, 15, 16]. Dineutron correlations are discussed also in light neutron-rich nuclei such as in ${ }^{8} \mathrm{He}[17,18,19]$ and also in medium-heavy neutron-rich nuclei [20, 21] as well as asymmetric nuclear matter (for example, Refs. 22, 23, 24, 25, 26, 27, 28, 29] and references therein).

Dineutron correlations are characterized by strong spatial correlations of two neutrons in the ${ }^{1} S$ channel. Although the neutron-neutron interaction is too weak to form a two-body bound state, the attraction in the ${ }^{1} S$ channel is rather strong as is known from the large scattering length $a_{s}=-18.5 \pm 0.4 \mathrm{fm}[30]$. Originally the possible existence of a dineutron near the surface of nuclei was predicted by Migdal in 1972 [31]. In his work, it was shown that two interacting particles in a potential well can form a bound state if there is a single-particle level with energy close to zero. This idea describes the binding mechanism of a Borromean system and is extended to the dineutron cluster picture and the dineutron correlation in two-neutron halo nuclei.

Recently, dineutron correlations have also been discussed from the point of view of Bose-Einstein condensation (BEC), which is considered along similar lines as deuteron and $\alpha$-particle condensation suggested in dilute nuclear matter [23, 32, 33, 34, 35] as well as $\alpha$ condensate states in excited states of $Z=N=$ even nuclei [36, 37, 38]. This $n n$-BEC feature stems from the surprisingly small size of Cooper pairs recently revealed in the surface of nuclei [21]. In nuclear systems, the $S=0 T=1$ pair correlation in the Bardeen-Cooper-Schrieffer (BCS) phase has been known to be very important [39, 40]. From the analysis of the spatial structure of the Cooper pair in neutron matter, a size shrinking is found to occur at finite low density [24, 27, 28]. It indicates that the spatially correlated neutron pair may appear in the transition region from weak coupling BCS regime to strong coupling BEC regime. Similar BCS-BEC crossover features of the spatially correlated neutron pair were predicted also at the surface of medium-heavy nuclei [20, 21]. Since nuclear pairing increases as the size of nuclei shrinks $(\Delta \sim 12 / \sqrt{A} \mathrm{MeV})$, one may expect that the BEC features are enhanced with respect to the infinite matter case.

Thus, the spatial structure of neutron pairs at the surface of neutron-rich nuclei attracts presently great interest. In particular, BCS-BEC cross over features are expected to appear in the environment of dilute neutron matter, such as realized in neutron skins [41, 42, 43]. This has been put into evidence in recent studies [20, 21] where it was found that the extension of a $n n$-Cooper pair strongly decreases when going from inside to the surface before expanding again when leaving the nucleus. The minimum is very pronounced and the Cooper pair attains a minimum size of about 2-3fm. This is very small size reaching the dimension of the deuteron, that is of a bound state. This is also 
highlighted by the fact that in ${ }^{11} \mathrm{Li}$ even the single Cooper pair is very small in the surface [44].

The fact that $n n$-Cooper pairs resemble a bound state in the nuclear surface and even become appreciably smaller than in low density neutron matter with maximum gap value, is intriguing. In this work we want to explore the rather extreme hypothesis that the binding mechanism may be influenced by some quasi two-dimensional features. By 'quasi two-dimensional (2D)', we mean that the 'bound' Cooper pairs are confined within a surface layer of about $1-2 \mathrm{fm}$ and that the degree of freedom in radial direction is approximately frozen. Such a scenario could for example be realized by the fact that the density distribution of valence neutrons in very neutron rich and heavy nuclei are in radial, say $z$-direction, concentrated in a surface layer and that the density distribution in $z$-direction can be approximated by a frozen Gaussian packet, whereas the motion of the neutrons within the layer is free. Pursuing the picture to its extreme, one could imagine a slab of low density neutrons where in the transverse $(z)$ direction only a single $0 s$ level below the Fermi energy is active. Such situation also could eventually be relevant in the Lasagne phase in neutron star crusts, where a low-density neutron gas may appear as a layer on the surface of the Lasagne structure [45, 46, 47, 48]. In any case, one could imagine that in the outer layer of a neutron-rich nucleus, the pairing properties of the valence neutrons are more or less decoupled from the inert core part. For example, very neutron-rich Sn isotopes with $N>82$ could represent such a scenario. A 'quasi-2D' slab of low density neutron matter with the transverse degrees of freedom approximately frozen could then, as already mentioned, mimic the situation. We here insist on the $2 \mathrm{D}$ character because it is well known that the dimensionality has great influence on bound state formation and, thus, also on the formation of tight Cooper pairs. Such quasi-2D paired systems with low density also have been investigated in cold atom physics e.g. hydrogen atoms on a liquid ${ }^{4} \mathrm{He}$ surface and laser cooled atoms trapped in an planar potential [49, 50].

At any rate, and for the different reasons mentioned above, it seemed to us interesting to study an idealized situation of quasi-2D low density neutron matter realized within an infinitely extended slab. The study may turn out academic in the end but if future more detailed and not completely trivial investigations will exhibit a link to the surprisingly strong bound state features of Cooper pairs in the nuclear surface, mentioned above, our effort will have been worth while. In this respect it is of interest to mention that, indeed, our study will show that in 2D matter a Cooper pair attains a minimal size of about $2 \mathrm{fm}$ whereas in 3D this value is over twice as large.

In the present work, we investigate properties of neutron pairs in quasi-2D neutron systems by using finite-range effective nuclear forces. Concerning the degree of freedom in the transverse $z$ direction perpendicular to the 2D plane, we for simplicity assume a Gaussian packet as mentioned above. We study the two-neutron wave function in this model of quasi-2D system and show that two neutrons form a tightly bound state with a small size when the width $a$ of the Gaussian in $z$-direction is small enough. We also investigate pairing properties of neutron matter based on the BCS theory. Pairing gap and size of the Cooper pair in quasi-2D neutron systems are analyzed, and BCS-BEC crossover phenomena are discussed.

The behaviour of $T=1$ pairing in infinite three-dimensional (3D) matter has been extensively investigated based on the BCS theory [51] for symmetric matter and neutron matter (for example, Refs. 222, 23, 25, 26] and references therein). As for a slab of nuclear matter, properties of $T=1$ pairing in symmetric nuclear matter have been studied in Refs. [52, 53, 54, 55] and it was shown that the pairing gap increases at the surface of the slab. As for $T=0$ pairing, the gradual transition from BCS to BEC involving the deuteron formation was predicted in dilute symmetric matter 23, 32, 33, 35, 56]. Competition of alpha and deuteron condensation was also discussed [34].

There are many varieties of BCS calculations of nuclear matter concerning, e.g., the treatment of single-particle spectra and the pairing interaction. For convenience, effective nuclear forces have been used in some calculations on pairing in nuclear matter instead of dealing with realistic bare nuclear forces. It was shown that the Gogny force [57, 58], a finite-range effective nuclear force, gives similar behaviour of nuclear matter pairing to that calculated from realistic nuclear forces with the Brueckner-HF (BHF) approximation, as well as reasonable pairing properties in finite nuclei 24, 57, 58, 59, 60]. Therefore, we adopt the Gogny D1S force in the present BCS calculations. We also use the Minnesota force [61] which reproduces features of $S$-wave $N-N$ scattering in $T=0$ and $T=1$ channels, and nucleus-nucleus scattering in the light nuclear region such as the $\alpha$ - $\alpha$ system, as well as properties of light nuclei. The Minnesota force is often used in structure studies of light neutron-rich nuclei.

This paper is organized as follows. In the next section, we explain our simplified model of quasi-2D systems and formulation of the present work. The effective nuclear forces are described in section IIII In section IV] the results obtained for a two-neutron system and neutron matter are shown, and in section $\mathbf{V}$ discussions are outlined. Finally, we give a summary and outlook in section VI.

\section{FORMULATION OF NUCLEAR 2D PAIRING}

Here we explain our framework of the simplified model of quasi-2D neutron system confined in a slab with a certain thickness. We first describe the wave function and the Hamiltonian for a quasi-2D neutron matter system. We then 
explain the isolated two-neutron system and give the formalism for the quasi-2D system within the BCS theory.

\section{A. Quasi-2D neutron systems}

According to the outline of the Introduction, we propose a model for a system of neutrons confined in a slab with a certain thickness. We assume that the neutrons in transverse $(z)$ direction are represented by a simple Gaussian packet of size $a$. As already mentioned, this size may mimic the concentration of the amplitudes of single particle wave functions in a surface layer, where $z$ corresponds to the radial direction in finite neutron-rich nuclei.

Therefore, the wave function for a single neutron is written as

$$
\begin{gathered}
\Phi(\mathbf{r})=\Phi^{2 D}\left(\mathbf{r}_{\perp}, \chi\right) \otimes \phi^{0 s}(z), \\
\phi^{0 s}(z)=\left(\frac{1}{\pi a^{2}}\right)^{1 / 4} \exp \left[-\frac{z^{2}}{2 a^{2}}\right],
\end{gathered}
$$

where $\mathbf{r}_{\perp}$ indicates the coordinates in the slab, $(x, y)$, and $\chi$ is the intrinsic spin. The thickness, i.e., the rms width, of the slab is $2 \sqrt{\left\langle z^{2}\right\rangle}=\sqrt{2} a$. In a similar way, a $N$-neutron wave function is given as

$$
\Phi\left(\mathbf{r}_{1}, \cdots, \mathbf{r}_{\mathbf{N}}, \chi_{1}, \cdots, \chi_{N}\right)=\Phi^{2 D}\left(\mathbf{r}_{1 \perp}, \cdots, \mathbf{r}_{N \perp}, \chi_{1}, \cdots, \chi_{N}\right) \otimes \phi^{0 s}\left(z_{1}\right) \cdots \phi^{0 s}\left(z_{N}\right) .
$$

Here $\Phi$ and $\Phi^{2 D}$ are the antisymmetrized many-body wave functions.

Neutrons are interacting with each other via two-body nuclear forces. The interaction $V\left(r_{i j}\right)$ between two neutrons is the two-body effective nuclear force in the $T=1$ channel. Here $r_{i j}=\left|\mathbf{r}_{\mathbf{i}}-\mathbf{r}_{\mathbf{j}}\right|$. Because the $S$-wave neutron pair correlation is studied in the present work, only the central part of the effective nuclear force is considered. The Gogny and Minnesota forces are represented by a superposition of two Gaussians,

$$
V(r)=\sum_{m=1}^{2}\left(W_{m}+B_{m} P_{\sigma}-H_{m} P_{\tau}-M_{m} P_{\sigma} P_{\tau}\right) \exp \left[-\frac{r^{2}}{b_{m}^{2}}\right]
$$

We freeze the the degree of freedom in transverse $(z)$ direction, as described in the Introduction. By integrating over $z_{i}$ coordinates the three-dimensional Schrödinger equation for $\Phi$ is reduced to the two-dimensional equation for $\Phi^{2 D}$ with respect to $\mathbf{r}_{i \perp}$. Then we get the following equation for quasi-2D neutron systems,

$$
\begin{gathered}
H^{2 D} \Phi^{2 D}\left(\mathbf{r}_{1 \perp}, \cdots, \mathbf{r}_{N \perp}, \chi_{1}, \cdots, \chi_{N}\right)=E^{2 D} \Phi^{2 D}\left(\mathbf{r}_{1 \perp}, \cdots, \mathbf{r}_{N \perp}, \chi_{1}, \cdots, \chi_{N}\right), \\
H^{2 D}=\sum_{i} t_{i \perp}+\sum_{i<j} V^{2 D}\left(r_{i j \perp}\right),
\end{gathered}
$$

where the 2D-kinetic term and the 2D-interaction term are

$$
\begin{aligned}
t_{\perp}=-\frac{\hbar^{2}}{2 m} \frac{\partial^{2}}{\partial \mathbf{r}_{\perp}^{2}}=-\frac{\hbar^{2}}{2 m}\left(\frac{\partial^{2}}{\partial x^{2}}+\frac{\partial^{2}}{\partial y^{2}}\right) \\
V^{2 D}\left(r_{\perp}\right)=\left\langle\phi^{0 s}\left(z_{1}\right) \phi^{0 s}\left(z_{2}\right)|V(r)| \phi^{0 s}\left(z_{1}\right) \phi^{0 s}\left(z_{2}\right)\right\rangle \\
=\sum_{m=1}^{2} Z_{b_{m}}(a)\left(W_{m}+B_{m} P_{\sigma}-H_{m} P_{\tau}-M_{m} P_{\sigma} P_{\tau}\right) \exp \left[-\frac{r_{\perp}^{2}}{b_{m}^{2}}\right] \\
Z_{b_{m}}(a) \equiv\left(\frac{b_{m}^{2}}{b_{m}^{2}+2 a^{2}}\right)^{1 / 2} .
\end{aligned}
$$

As seen from (8) and (9), the strength of the two-body interaction $V^{2 D}\left(r_{\perp}\right)$ in the quasi-2D system depends on the thickness of the slab with the factor $Z_{b_{m}}(a)$. The factor decreases with the increase of the width parameter $a$. The thickness of the slab is only reflected in a weakening of the interaction for bigger slab sizes in our genuinely two-dimensional model. These are, of course, strongly simplified, may be oversimplified, assumptions to describe the detailed behaviour of the valence neutrons because the Pauli effect from the core should be more complicated and a long tail of weakly bound neutrons may be not so simple. However, it is expected that such effects may effectively be taken into account by a modification of the width parameter $a$. In the present work, we analyze properties of neutron pairs in quasi-2D neutron systems for various values of the slab size $a$. 


\section{B. The isolated two-neutron system}

For later comparison we consider the $S$-wave relative wave function $\Phi^{n n}\left(r_{\perp}, \chi_{1}, \chi_{2}\right)$ for an isolated two-neutron system in the quasi-2D space. It is obtained by solving the Schrödinger equation,

$$
\begin{aligned}
& \left(-\frac{\hbar^{2}}{m}\left(\frac{\partial^{2}}{\partial r_{\perp}}+\frac{1}{r_{\perp}} \frac{\partial}{\partial r_{\perp}}\right)+V^{2 D}\left(r_{\perp}\right)\right) \Phi^{n n}\left(r_{\perp}, \chi_{1}, \chi_{2}\right)=E_{n n}^{2 D} \Phi^{n n}\left(r_{\perp}, \chi_{1}, \chi_{2}\right), \\
& \Phi^{n n}=\psi^{n n}\left(r_{\perp}\right) \otimes X_{S=0}\left(\chi_{1}, \chi_{2}\right) .
\end{aligned}
$$

$\psi^{n n}\left(r_{\perp}\right)$ is the spatial wave function normalized as $\int d^{2} r_{\perp}\left|\psi^{n n}\left(r_{\perp}\right)\right|^{2}=1$, and $X_{S=0}$ is the intrinsic-spin wave function for the spin-zero channel.

It is important to note that in two dimensions, a bound state can form at any arbitrarily small attraction [62]. Since the nuclear force in the ${ }^{1} S$ channel is attractive at low energy, a two-neutron bound state in the quasi-2D space is obtained with an arbitrary width $a$. We calculate the exact solution of the bound state with a Gaussian expansion method. The size of the two-neutron bound state is given as

$$
\xi_{\perp}^{n n}=\sqrt{\int d^{2} r_{\perp} r_{\perp}^{2}\left|\psi^{n n}\left(r_{\perp}\right)\right|^{2}}
$$

Please note that the size in $3 \mathrm{D}$ is given as $\xi=\sqrt{\left(\xi_{\perp}^{n n}\right)^{2}+a^{2}}$.

\section{Quasi two-dimensional neutron matter within BCS theory}

In this section, we investigate the behaviour of neutron pairs in quasi-2D infinite neutron matter in the slab by means of the BCS theory, which is equivalent to the HFB approximation in a homogeneous case [39, 40]. The application of BCS theory with the Gogny force to a slab of nuclear matter was, e.g., already performed in Ref. [53].

Let us explain the equations for ${ }^{1} S$ pairing in quasi-2D neutron matter. We apply the BCS theory to the $2 \mathrm{D}$ Schrödinger equation (5). The $S$-wave pairing gap depends on $\left|\mathbf{p}_{\perp}\right|$ and it is written as

$$
\begin{gathered}
\Delta(p)=-\frac{1}{2} \int \frac{d^{2} k_{\perp}}{(2 \pi)^{2}} v_{\mathrm{pp}}\left(\mathbf{p}_{\perp}-\mathbf{k}_{\perp}\right) \frac{\Delta(k)}{\sqrt{(e(k)-\mu)^{2}+\Delta(k)^{2}}}, \\
e(p)=\frac{\mathbf{p}_{\perp}^{2}}{2 m}+V^{H F}(p) .
\end{gathered}
$$

Here $p$ and $k$ denote $\left|\mathbf{p}_{\perp}\right|$ and $\left|\mathbf{k}_{\perp}\right|$, respectively, and $v_{\mathrm{pp}}$ is the pairing force,

$$
v_{\mathrm{pp}}\left(\mathbf{p}_{\perp}-\mathbf{k}_{\perp}\right)=\sum_{m=1}^{2} Z_{b_{m}}(a)\left(W_{m}-B_{m}-H_{m}+M_{m}\right)\left(\sqrt{\pi} b_{m}\right)^{2} \exp \left[-\frac{1}{4} b_{m}^{2}\left(\mathbf{p}_{\perp}-\mathbf{k}_{\perp}\right)^{2}\right] .
$$

$V^{H F}(p)$ is the Hartree-Fock potential,

$$
V^{H F}(p)=\frac{\rho}{2} \sum_{m=1}^{2} Z_{b_{m}}(a)\left(2 W_{m}+B_{m}-2 H_{m}-M_{m}\right)\left(\sqrt{\pi} b_{m}\right)^{2}-\int \frac{d^{2} k_{\perp}}{(2 \pi)^{2}} v_{\mathrm{ph}}\left(\mathbf{p}_{\perp}-\mathbf{k}_{\perp}\right) v_{k}^{2}
$$

where the exchange term is given as

$$
v_{\mathrm{ph}}\left(\mathbf{p}_{\perp}-\mathbf{k}_{\perp}\right)=\sum_{m=1}^{2} Z_{b_{m}}(a)\left(W_{m}+2 B_{m}-H_{m}-2 M_{m}\right)\left(\sqrt{\pi} b_{m}\right)^{2} \exp \left[-\frac{1}{4} b_{m}^{2}\left(\mathbf{p}_{\perp}-\mathbf{k}_{\perp}\right)^{2}\right] .
$$

The occupation probability is

$$
v_{k}^{2}=\frac{1}{2}\left(1-\frac{e(k)-\mu}{\sqrt{(e(k)-\mu)^{2}+\Delta(k)^{2}}}\right)
$$


and the chemical potential $\mu$ is determined so as to satisfy the number equation,

$$
\frac{\rho}{2}=\frac{k_{F}^{2}}{4 \pi}=\int \frac{d^{2} k_{\perp}}{(2 \pi)^{2}} v_{k}^{2}
$$

where $\rho$ and $k_{F}$ are the density and the Fermi momentum in 2D, respectively. In this paper, the $k_{F}$ dependence of pairing properties in neutron matter is discussed as a function of the ratio $k_{F} / k_{0}$, where $k_{0}=1.36 \mathrm{fm}{ }^{-1}$ is the Fermi momentum at the normal density of 3D symmetric nuclear matter.

The energy per nucleon for the BCS state is

$$
E_{B C S} \equiv \frac{1}{\rho}\left\langle\Phi_{\mathrm{BCS}}\left|H^{2 D}\right| \Phi_{\mathrm{BCS}}\right\rangle=\frac{1}{\rho} \int \frac{d^{2} k_{\perp}}{(2 \pi)^{2}}\left[\frac{\mathbf{p}_{\perp}^{2}}{2 m}+\frac{1}{2} V^{H F}(k)\right] 2 v_{k}^{2}+E_{\text {pair }}
$$

where the integrated pair energy $E_{\text {pair }}$ is

$$
E_{\text {pair }} \equiv-\frac{1}{\rho} \int \frac{d^{2} k_{\perp}}{(2 \pi)^{2}} \Delta(k) \kappa(k)
$$

with

$$
\kappa(k)=u_{k} v_{k}=\frac{1}{2} \frac{\Delta(k)}{\sqrt{(e(k)-\mu)^{2}+\Delta(k)^{2}}} .
$$

The energy per nucleon for the HF state $\Phi_{\mathrm{HF}}$ is determined from

$$
E_{H F} \equiv \frac{1}{\rho}\left\langle\Phi_{\mathrm{HF}}\left|H^{2 D}\right| \Phi_{\mathrm{HF}}\right\rangle
$$

To analyze the spatial structure of a $n n$-Cooper pair it is useful to study the pair wave function in the coordinate space. It is defined by the Fourier transform of the anomalous density $\kappa(k)$,

$$
\Psi_{\text {pair }}\left(r_{\perp}\right) \equiv n_{0}\left\langle\Phi_{\mathrm{BCS}}\left|a^{\dagger}\left(\mathbf{r}_{\perp}^{\prime} \uparrow\right) a^{\dagger}\left(\mathbf{r}_{\perp}^{\prime \prime} \downarrow\right)\right| \Phi_{\mathrm{BCS}}\right\rangle=n_{0} \int \frac{d^{2} k_{\perp}}{(2 \pi)^{2}} \kappa(k) e^{i \mathbf{k}_{\perp} \cdot\left(\mathbf{r}_{\perp}^{\prime}-\mathbf{r}_{\perp}^{\prime \prime}\right)} .
$$

Here $r_{\perp}=\left|\mathbf{r}_{\perp}^{\prime}-\mathbf{r}_{\perp}^{\prime \prime}\right|$, and $n_{0}$ is the normalization factor so that $\int d^{2} r_{\perp}\left|\Psi_{\text {pair }}\left(r_{\perp}\right)\right|^{2}=1$. The pair size (coherence length) $\xi_{\perp}$ is calculated from the root-mean-square distance of the pair wave function

$$
\xi_{\perp} \equiv \sqrt{\left\langle r_{\perp}^{2}\right\rangle}
$$

where

$$
\left\langle r_{\perp}^{2}\right\rangle=\int d^{2} r_{\perp} r_{\perp}^{2}\left|\Psi_{\text {pair }}\left(r_{\perp}\right)\right|^{2}
$$

In the low-density limit $k_{f} \rightarrow 0$, the pair wave function $\Psi_{\text {pair }}$ and the energy $2 E_{B C S}$ approach $\psi^{n n}$ and $E_{n n}^{2 D}$ of the two-neutron bound state of section II.B.

\section{EFFECTIVE NUCLEAR FORCES AND PAIRING PROPERTIES IN 3D NEUTRON MATTER}

As already mentioned, in this work we use the Gogny D1S force [57, 58] and the Minnesota force [61]. In the $T=1$ channel, the Gogny force is density independent and is represented by a superposition of two Gaussians (Eq. (4)). The parameters for the Gogny D1S force are,

$$
\begin{aligned}
& b_{1}=0.7 \mathrm{fm}, \quad b_{2}=1.2 \mathrm{fm} \\
& W_{1}=-1720.30 \mathrm{MeV}, \quad B_{1}=1300.00 \mathrm{MeV} \\
& H_{1}=-1813.53 \mathrm{MeV}, \quad M_{1}=1397.60 \mathrm{MeV} \\
& W_{2}=103.64 \mathrm{MeV}, \quad B_{2}=-163.48 \mathrm{MeV} \\
& H_{2}=162.81 \mathrm{MeV}, \quad M_{2}=-223.93 \mathrm{MeV} .
\end{aligned}
$$


The Minnesota force is also given by a sum of finite-range Gaussians,

$$
\begin{aligned}
V(r)= & \frac{1}{2}\left(1-P_{\sigma}\right) \frac{1}{2}\left(1+u P_{r}\right)\left(V_{R} \exp \left(-\kappa_{R} r^{2}\right)+V_{s} \exp \left(-\kappa_{s} r^{2}\right)\right) \\
& +\frac{1}{2}\left(1+P_{\sigma}\right) \frac{1}{2}\left(1+u P_{r}\right)\left(V_{R} \exp \left(-\kappa_{R} r^{2}\right)+V_{t} \exp \left(-\kappa_{t} r^{2}\right)\right), \\
V_{R}= & 200.0 \mathrm{MeV}, \kappa_{R}=1.487 \mathrm{fm}^{-2}, \\
V_{s}= & -91.85 \mathrm{MeV}, \kappa_{s}=0.465 \mathrm{fm}^{-2}, \\
V_{t}= & -178.0 \mathrm{MeV}, \kappa_{t}=0.639 \mathrm{fm}^{-2} .
\end{aligned}
$$

We adopt for the exchange-mixture parameter the value of $u=1.0$. Then the first (second) term gives the two-body force in the $S=0 T=1(S=1 T=0)$ channel.

In principle the Gogny force represents an effective in medium force and the Minnesota force a phenomenological nuclear force in free space. It has, however, been shown that the Gogny D1S force gives similar behaviour for the ${ }^{1} S$ pairing gap of symmetric nuclear matter as that calculated from realistic nuclear forces with the BHF+BCS approximation, and also describes pairing properties in finite nuclei [24, 57, [58, 59, 60]. The Minnesota force reasonably reproduces the $S$-wave $N-N$ scattering in the $S=0 T=1$ channel as well as the $S=1 T=0$ channel. It also well describes nucleus-nucleus scattering such as the $\alpha-\alpha$ system, as well as properties of light nuclei. Because the Minnesota force has no density-dependent term in the $S=1 T=0$ channel, it can not describe the saturation properties of symmetric nuclear matter. However, it gives a similar density dependence of neutron matter energy as the one obtained with the Gogny D1S force, as shown later.

The values of the $n$ - $n$ scattering length $a_{s}$ in the ${ }^{1} S$ channel obtained with the Gogny D1S force and Minnesota force are $a_{s}=-12.1 \mathrm{fm}$ and $a_{s}=-16.8 \mathrm{fm}$, respectively. These values semiquantitatively agree with the experimental value $a_{s}=-18.5 \pm 0.4 \mathrm{fm}$ [30]. In Fig. 1] the calculated phase shifts of $N$ - $N$ scattering in the ${ }^{1} S$ channel are shown as well as those obtained with a realistic force. Both the Gogny D1S and Minnesota forces show a reasonable reproduction of the ${ }^{1} S$-wave scattering.

Figure 2 shows the properties of infinite 3D neutron matter calculated with the Gogny D1S and Minnesota forces by means of the BCS theory as well as from the HF calculations. Figures 2 (a) and 2(b) show the the energy $\left(E_{B C S}\right)$ and the integrated pair energy $\left(E_{\text {pair }}\right)$ per nucleon, and the chemical potential $(\mu)$ of the BCS state are shown as well as the energy of the HF state $\left(E_{H F}\right)$ for 3D neutron matter with the Gogny D1S and Minnesota forces, respectively. The density dependence of the neutron matter energy obtained with both forces is in reasonable agreement with that calculated with ab initio methods using realistic nuclear forces [63, 64].

In Fig. 2(c), the pairing gap of neutron matter is shown. The difference between the Gogny D1S and Minnesota forces is not large in the low-density region $k_{F} / k_{0}<0.4$. In the $k_{F} / k_{0} \geq 0.4$ region, the Minnesota force gives a much smaller pairing gap than the Gogny D1S force because it has a larger repulsive core with $108.15 \mathrm{MeV}$ hight at $r=0$.

Let us make a comment on the pairing gap in 3D neutron matter investigated in different approaches. There are many calculations of the pairing gap in nuclear matter using realistic nuclear forces (for reviews [23, 25]). In the standard $\mathrm{BHF}+\mathrm{BCS}$ approximation, the pairing gap in 3D neutron matter is about $3 \mathrm{MeV}$ at the peak position $k_{f} / k_{0} \sim 0.6$ (figure 12 of Ref. [25]), which is consistent with the pairing gap calculated in BCS theory with the Gogny force. In different approximations with medium polarization effects, a quenching of the pairing gap in neutron matter was suggested [28, 65, 66, 67, 68, 69].

The size $\xi$ of a Cooper pair in 3D neutron matter is plotted as a function of $k_{F} / k_{0}$ in Fig. 2(d). As is already discussed in Refs. [24, 27, 28], the pair size shrinking in 3D neutron matter is seen at finite low $k_{F}$. Similarly to the pairing gap, the difference of the pair size between the Gogny and Minnesota forces is small in the low-density region. In the $k_{F} / k_{0} \geq 0.4$ region, the pair size increases more rapidly because of the weaker pairing with the Minnesota force than is the case with the Gogny D1S force. As we show later, also in quasi-2D neutron matter these two forces give qualitatively similar pairing behaviour for low $k_{F}$. With both forces, the $\xi<d$ region ( $d$ is the average inter-neutron distance $d=\rho^{-1 / 3}$ ) appears at finite low $k_{F}$, i.e., at low density. It shows the BCS-BEC crossover feature as suggested by Matsuo [27].

\section{RESULTS}

In this section, two-neutron correlations in quasi-2D neutron systems are investigated by performing BCS calculations for 2D neutron matter with the Gogny D1S and Minnesota forces. The results for various values of $a$ of the confinement in the frozen direction $(z)$ are analyzed, and the behaviour of the Cooper pair in quasi-2D neutron matter is discussed as a function of $k_{F} / k_{0}$. 


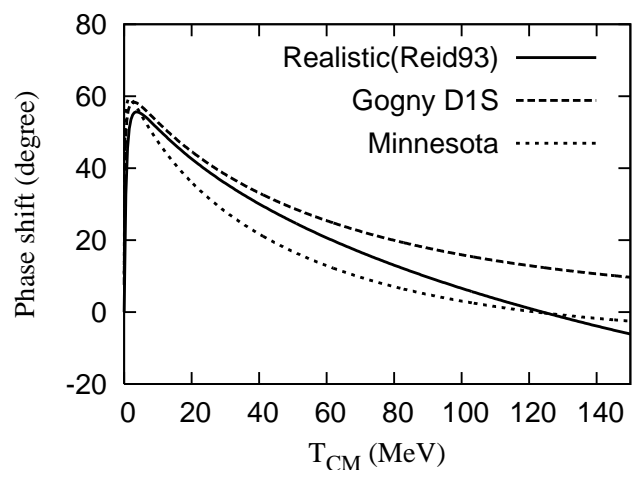

FIG. 1: Phase shift of $N-N$ scattering in the ${ }^{1} S$ channel obtained with the Gogny D1S force and Minnesota force. ${ }^{1} S$ phase shift of a realistic force, Reid93 potential [70], which fits the experimental proton-proton scattering cross sections, is also shown.

\section{A. Two-neutron bound state in quasi-2D system}

By solving the Schrödinger equation (10), the wave function $\psi^{n n}\left(r_{\perp}\right)$ of two isolated neutrons in a quasi-2D system is obtained. While a two-body bound state in 3D forms only at sufficiently strong attraction, a bound state in 2D can form, as already mentioned, at any arbitrarily small attraction. Since the ${ }^{1} S$ nuclear force is attractive at low energy, the two-neutron bound state is formed in quasi-2D systems with arbitrary width $a$.

The energy $E_{n n}^{2 D}$ and the pair size $\xi_{\perp}^{n n}$ of the bound state for $a=1, a=2$ and $a=4 \mathrm{fm}$ are given in Table [. Energy and size of the dineutron decrease as the width $a$ becomes small. In the quasi-2D system with a small width of $a=1 \mathrm{fm}$, the dineutron size is as small as that of the deuteron in 3D. On the other hand, in the quasi-2D system with a width $a=4 \mathrm{fm}$, the dineutron has a small binding energy, less than $0.1 \mathrm{MeV}$, and a size larger than $20 \mathrm{fm}$. We, therefore, see that in our model the width of the slab has a strong influence on the two body correlations.

It is very interesting that, although two neutrons are unbound in $3 \mathrm{D}$, they form a tightly bound dineutron state with a size comparable to deuteron dimensions when they are confined in a sufficiently thin slab. To form the small-sized dineutron, the reduction of the dimension from three to two is crucial. In the present model, neutrons are confined in a Gaussian packet with respect to the frozen $z$-direction.

In slab direction, the potential part $V^{2 D}\left(r_{\perp}\right)$ is given by almost the same form as the original effective nuclear force $V(r)$ except for the factor $Z_{b_{m}}(a)$ in (8) . This factor is smaller than one, depending on $a$. For example, for the case of $a=1 \mathrm{fm}$ and the interaction range $b_{2}=1.2 \mathrm{fm}$ as with the long-range attractive term of the Gogny D1S force, one gets $Z_{b_{m}}(a)=0.59$, that is the strength of $V^{2 D}\left(r_{\perp}\right)$ is smaller than that in the original interaction $V(r)$. Let us remind the reader that the size of the dineutron state is determined by the balance of the kinetic energy and the potential energy. In a $2 \mathrm{D}$ system, the kinetic effect is weaker and a small-sized two-particle bound state can more easily be formed than in the $3 \mathrm{D}$ case.

In Fig. 3 the wave function $\psi^{n n}\left(r_{\perp}\right)$ and the probability density $r_{\perp}\left|\psi^{n n}\left(r_{\perp}\right)\right|^{2}$ of two isolated neutrons in a quasi$2 \mathrm{D}$ system are shown as a function of the relative coordinate $r_{\perp}$. In the quasi-2D system with $a=1$ and $a=2 \mathrm{fm}$, the probability density has a peak around $r_{\perp}=2 \mathrm{fm}$, which shows the significant spatial correlation between the neutrons. In a quasi-2D system with $a=4 \mathrm{fm}$, the density distributes in a broad region, and the spatial correlation is much weaker.

\section{B. Quasi-2D neutron matter}

We now extend the BCS calculations to quasi-2D neutron matter with varying width parameters $a$.

The pairing gap at the Fermi momentum $\Delta_{F} \equiv \Delta\left(k=k_{F}\right)$ as a function of $k_{F} / k_{0}$ is shown in Fig. 4, The Gogny D1S and Minnesota forces give qualitatively similar results in the low $k_{F}$ region. At $k_{F} / k_{0} \geq 0.3$, the pairing gap is more suppressed in case of the Minnesota force, because, as already mentioned, the short-range repulsion is larger than with the Gogny force. For $a=1 \mathrm{fm}$, the pairing gap obtained with the Gogny D1S force has a peak about 4 $\mathrm{MeV}$ high at $k_{F} / k_{0} \sim 0.4$, while that calculated with the Minnesota force has a peak $\sim 3 \mathrm{MeV}$ high at $k_{F} / k_{0} \sim 0.3$. With the increase of the width $a$ of the slab, the pairing gap $\Delta_{F}$ is quenched because the attraction of the pairing force is weaker in a wider slab. 

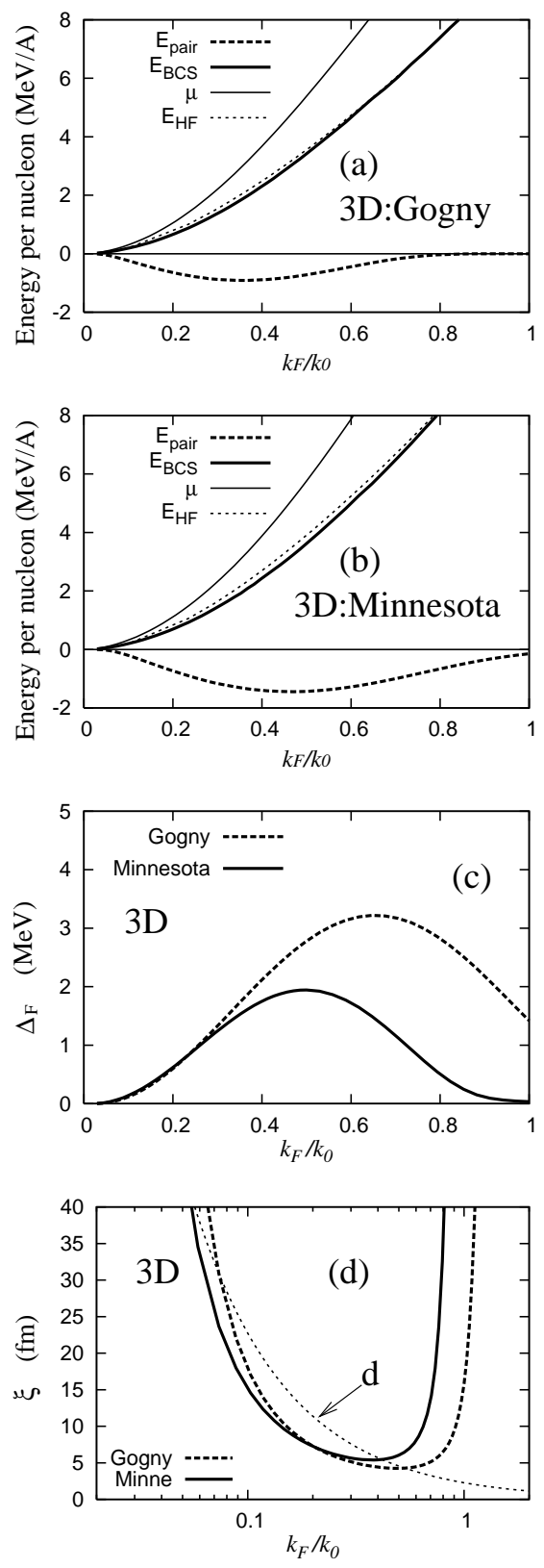

FIG. 2: Energy, pairing gap, chemical potential and pair size in 3D neutron matter as a function of $k_{F} / k_{0}\left(k_{0}=1.36\right.$ fm $\left.{ }^{-1}\right)$ obtained with the Gogny D1S force and Minnesota force. (a)(b) Energy $\left(E_{B C S}\right)$, the integrated pair energy $\left(E_{\text {pair }}\right)$ per nucleon and the chemical potential $(\mu)$ of the BCS state, and the energy of the HF state $\left(E_{H F}\right)$. (c) Gap energy at the Fermi momentum $\Delta_{F}=\Delta\left(k=k_{F}\right)$. (d) Pair size $\xi$. The average inter-neutron distance $d=\rho^{-1 / 3}$ is shown by a thin dashed line.

In Fig. 5, the energy $\left(E_{B C S}\right)$ and the integrated pair energy $\left(E_{\text {pair }}\right)$ per nucleon, and the chemical potential $(\mu)$ of the BCS state are shown as well as the energy of the HF state $\left(E_{H F}\right)$ calculated without pairing. Compared with the energy of 3D neutron matter shown in Fig. 2, one of the striking features of quasi-2D neutron matter is that $E_{B C S}$, $E_{\text {pair }}$, and the chemical potential have finite negative values even in $k_{F} \rightarrow 0$ limit. This is because, in the $k_{F} \rightarrow 0$ limit, the system goes to the strong coupling BEC limit, where Cooper pairs become identical with the two-particle bound state. In this limit, $-2 E_{B C S}$ and $-2 \mu$ become equal to the dineutron binding energy. As soon as the chemical potential turns negative, there is true binding between the two neutrons and the strong coupling BEC feature is born out. Therefore, in quasi-2D neutron matter one can, contrary to the 3D case, follow the transition from BEC to the BCS regime. On the other hand, because of the same reason, the pairing gap $\Delta_{F}$ in the quasi-2D neutron matter 
TABLE I: Energy $E_{n n}^{2 D}$ and size $\xi_{\perp}^{n n}$ of the two-neutron bound state $\psi^{n n}\left(r_{\perp}\right)$ in the quasi-2D system with the width parameter $a=1,2$, and $4 \mathrm{fm}$. The energy and the size $\xi^{p n}$ for a deuteron in 3D space obtained with the Minnesota force is also shown for comparison.

\begin{tabular}{cccc}
\hline & Gogny D1S \\
& $a=1 \quad a=2 \quad a=4$ \\
$E_{n n}^{2 D}(\mathrm{MeV})$ & -2.7 & -0.64 & -0.03 \\
$\xi_{\perp}^{n n}(\mathrm{fm})$ & 3.5 & 6.8 & 33 \\
\hline \multicolumn{4}{c}{ Minnesota } \\
& $a=1 \quad a=2 \quad a=4$ \\
$E_{n n}^{2 D}(\mathrm{MeV})$ & -1.9 & -0.74 & -0.06 \\
$\xi_{\perp}^{n n}(\mathrm{fm})$ & 4.3 & 6.5 & 21 \\
\hline \multicolumn{4}{c}{ Deuteron with Minnesota } \\
$E^{n}(\mathrm{MeV})$ \\
$\xi^{p n}(\mathrm{fm})$
\end{tabular}
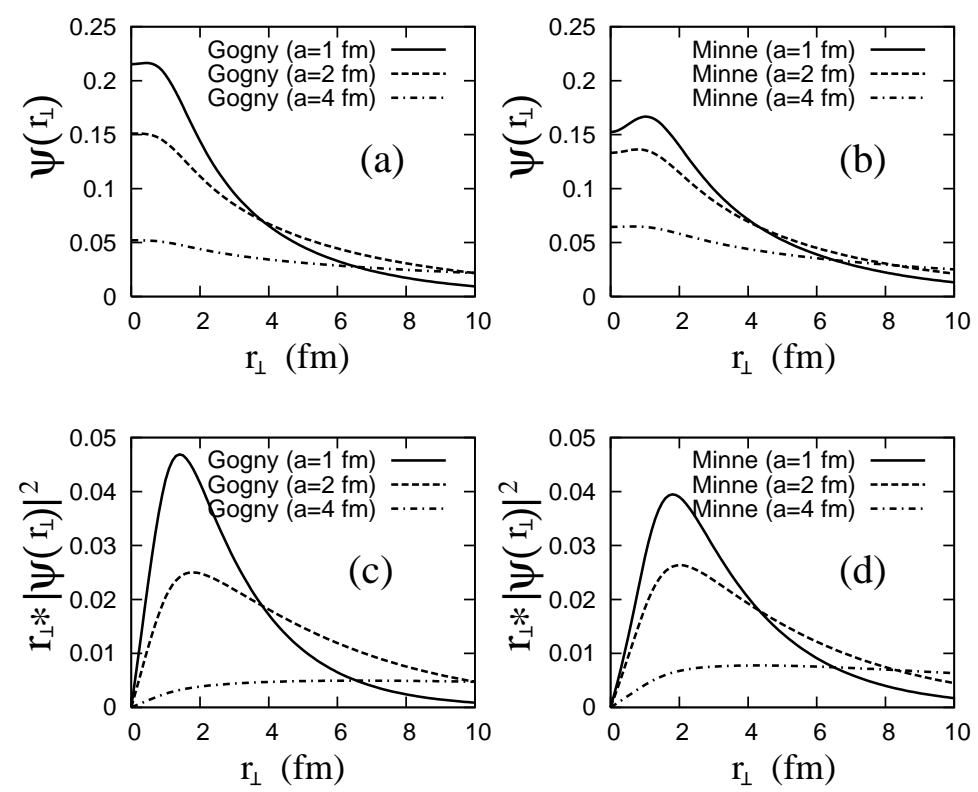

FIG. 3: Two-neutron wave function $\psi^{n n}\left(r_{\perp}\right)$ in quasi-2D systems with the width parameter $a=1,2$, and 4 fm calculated with (a) the Gogny D1S force and (b) Minnesota force. (c)(d) Same as (a)(b) but for the probability density $r_{\perp}\left|\psi^{n n}\left(r_{\perp}\right)\right|^{2}$.

shows a linear behaviour at the low $k_{F}$ limit (Fig. (4), while the pairing gap disappears with a horizontal tangent at small $k_{F}$ in 3D neutron matter (Fig. 2(c)).

The pair size $\xi_{\perp}$ in quasi-2D neutron matter is plotted as a function of $k_{F} / k_{0}$ in Fig. 6. In the $k_{F} \rightarrow 0$ limit, the pair size $\xi_{\perp}$ equals the size of the two-neutron bound state $\left(\xi_{\perp}^{n n}\right.$ in (12)). With the increase of $k_{F}$, the pair size reduces first before it becomes large again with further increase of $k_{F}$. Thus the minimum pair size $\xi_{\perp}$ is found to be smaller than the size $\xi_{\perp}^{n n}$ of the isolated two-neutron bound state in the quasi-2D system. This size reduction of the $n n$-Cooper pair indicates the enhancement of the dineutron correlation at finite low $k_{F}$, i.e. due to the existence of a Fermi sea. It should be pointed out that the pair size significantly depends also on the width $a$ of the slab.

A striking difference of the pair size between the quasi-2D and 3D neutron matter is that the pair size at $k_{F} \rightarrow 0$ limit is finite in the quasi-2D neutron matter while it becomes infinite in the 3D neutron matter. In the 3D neutron matter, the Cooper pair size $\xi$ has a minimum around $k_{F} / k_{0}=0.5$, and it rapidly increases as $k_{F}$ goes 0 . Interestingly, the size shrinking of the Cooper pair in quasi-2D neutron matter has a close analogy with that of the deuteron in 3D symmetric matter predicted by Lombardo and Schuck [56].

As mentioned above, the spatial correlation of Cooper pairs enhances at finite low $k_{F}$ in quasi-2D neutron matter 

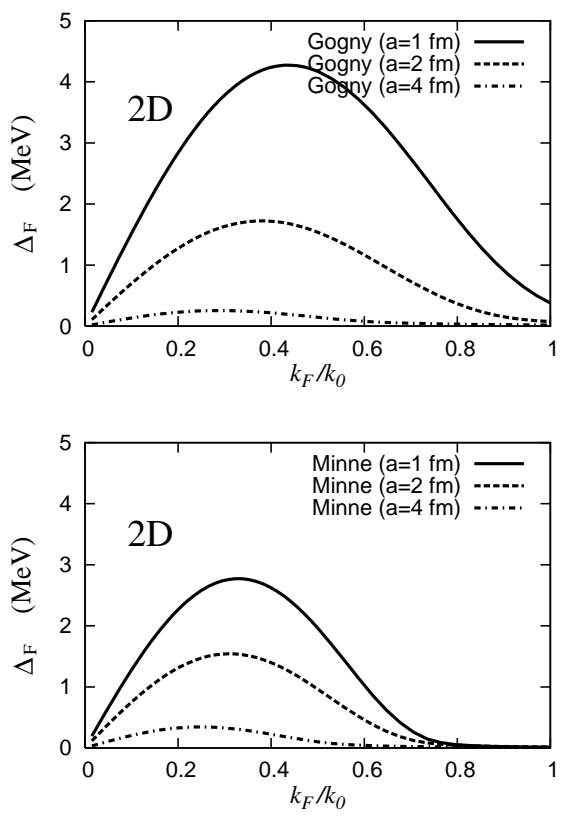

FIG. 4: Pairing gap at the Fermi momentum $\Delta_{F}=\Delta\left(k=k_{F}\right)$ in quasi-2D neutron matter as a function of $k_{F} / k_{0}\left(k_{0}=1.36\right.$ $\left.\mathrm{fm}^{-1}\right)$. Upper and lower panels show the results with the Gogny D1S force and Minnesota force, respectively.
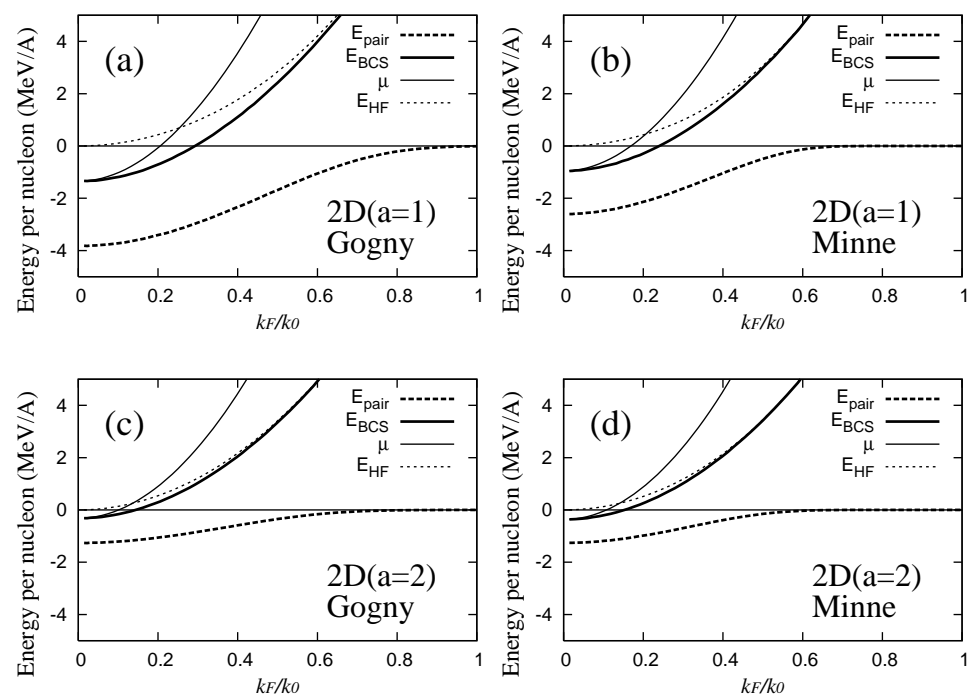

FIG. 5: Energy $\left(E_{B C S}\right)$, the integrated pair energy $\left(E_{\text {pair }}\right)$ per nucleon and the chemical potential $(\mu)$ of the BCS state, and energy of the HF state $\left(E_{H F}\right)$ as a function of $k_{F} / k_{0}\left(k_{0}=1.36 \mathrm{fm}^{-1}\right)$. (a) and (b): Energy of the quasi-2D neutron matter with $a=1 \mathrm{fm}$ obtained with the Gogny D1S force and Minnesota force. (c) and (d): Energy of the quasi-2D neutron matter with $a=2 \mathrm{fm}$.

with a small width $a$, where the BCS-BEC crossover phenomena is expected. To discuss pairing properties from the point of view of the BCS-BEC crossover, it is useful to compare the pair size $\xi_{\perp}$ with the average inter-neutron distance $d \equiv \rho^{-1 / 2}$ as discussed in the works for 3D neutron matter [27, 28]. The ratio $\xi_{\perp} / d$ in quasi-2D neutron matter is shown in Fig. 7 as well as $\xi / d$ for the 3D neutron matter. Strictly speaking, because the transition from the weak coupling BCS phase to strong coupling BEC is known to be a crossover [76], there is no critical boundary of the transition. However, we consider that BCS-BEC crossover features arise when the Cooper pair size is smaller than the average inter-neutron distance by adopting the same criterion as in Refs. [27, 28]. With this criterion, the BCS-BEC 

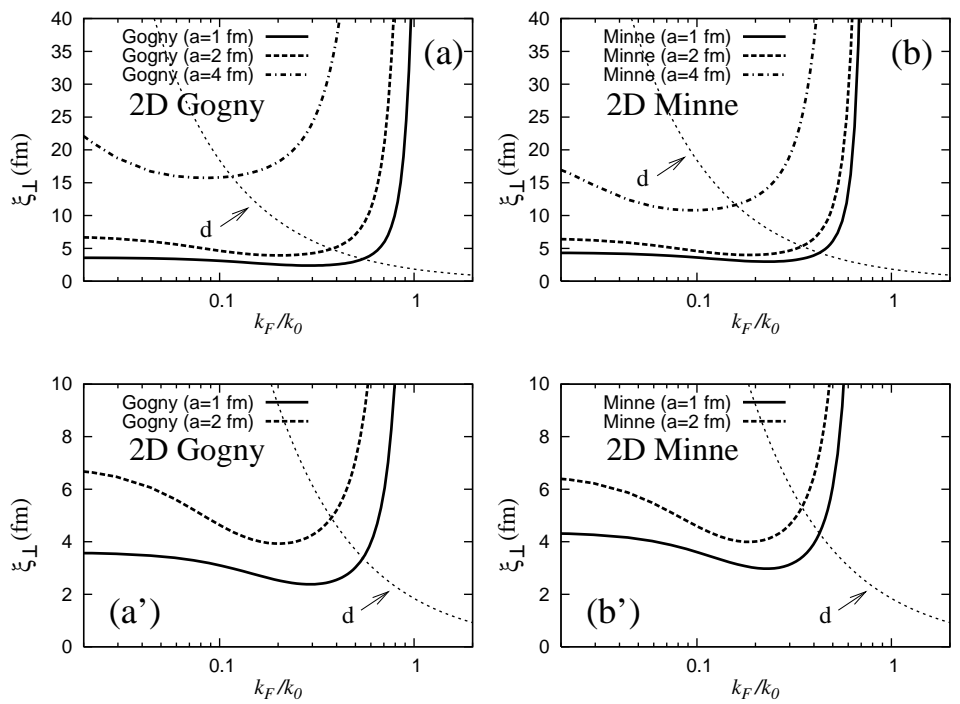

FIG. 6: (a)(b) Size $\xi_{\perp}$ of a Cooper pair $\Psi_{\text {pair }}$ in quasi-2D neutron matter obtained with the Gogny D1S and Minnesota forces. The calculated values are plotted as a function of $k_{F} / k_{0}\left(k_{0}=1.36 \mathrm{fm}^{-1}\right)$. The average inter-neutron distance $d=\rho^{-1 / 2}$ is plotted with the dotted lines. (a')(b') Same as (a)(b) but scaled up for the vertical axis.

crossover phenomena appears at $k_{F} / k_{0}<0.4 \sim 0.5$ in quasi-2D neutron matter with $a=1 \mathrm{fm}$, and $k_{F} / k_{0}<0.3 \sim 0.4$ for $a=2 \mathrm{fm}$, while in 3D neutron matter it is seen at $k_{F} / k_{0}<0.4 \sim 0.5$. In the quasi-2D neutron matter, the ratio $\xi_{\perp} / d$ monotonically deceases as $k_{F}$ becomes small, and finally the system goes to the strong coupling BEC limit.

To reveal the features of BCS-BEC crossover it is also useful to analyze the spatial structure of the $n n$-Cooper pair wave function [89] as done in Refs. 27, 28]. Figures 8] and 9] show the probability density $r_{\perp}\left|\Psi_{\text {pair }}\left(r_{\perp}\right)\right|^{2}$ and pair wave function $\Psi_{\text {pair }}\left(r_{\perp}\right)$ of the Cooper pair in the quasi-2D neutron matter. Let us discuss the results for $a=2 \mathrm{fm}$. At low $k_{F}$ such as $k_{F}=k_{0} / 8$ and $k_{F}=k_{0} / 4$ (Fig. 8 (b4) and (b3)), the spatial correlation of the Cooper pair is strong and it indicates a BEC-like dineutron pair. From $k_{F}=k_{0} / 8$ to $k_{F}=k_{0} / 4$, the first peak becomes narrow due to the Pauli principle, stemming from the other Cooper pairs. Consequently, the size shrinking of the dineutron occurs at a finite low $k_{F}$, as mentioned. With further increase of $k_{F}$, the nodal structure appears at $k_{F}=k_{0} / 2$ which indicates the transition from the BCS-BEC crossover to the BCS regime, and at $k_{F}=k_{0}$ the short-range correlation disappears as in the weak coupling BCS phase.

The pair wave functions in quasi-2D neutron matter with $a=2 \mathrm{fm}$ are shown in Fig. 9. We also show the twoneutron wave function $\psi^{n n}\left(r_{\perp}\right)$ for the dineutron bound state in the quasi-2D system and $\Psi_{\text {pair }}(r)$ in 3D neutron matter. In the $k_{F} \rightarrow 0$ limit, the pair wave function $\Psi_{\text {pair }}\left(r_{\perp}\right)$ becomes equal to the isolated two-neutron wave function $\psi^{n n}\left(r_{\perp}\right)$ of the dineutron state in case of quasi-2D neutron matter, while $\Psi_{\text {pair }}(r)$ in 3D neutron matter becomes infinitely broad because there is no two-neutron bound state in a $3 \mathrm{D}$ system.

\section{DISCUSSION}

\section{A. Possible relation to neutron pair correlations in neutron skins}

In this section we tentatively associate the strong pairing correlations of valence neutrons in neutron-rich nuclei with the ones in a $2 \mathrm{D}$ system. It has recently become evident that in neutron-rich or neutron halo nuclei pairing correlations may show a strong maximum in the surface. This is for instance evidenced by the fact that the size of the Cooper pairs has a sharp minimum in the surface. This feature has been shown to hold true in the case of a single Cooper pair [77] as well as in cases of many Cooper pairs [21]. Though for the moment, it cannot be excluded that the pronounced small Cooper pair sizes in the nuclear surface is due to a somewhat trivial, i.e. geometrical effect [78], we here make the hypothesis that this is due to a real strong correlation effect, due to the presence of a surface and hence of a $2 \mathrm{D}$ influence.

The valence nucleons may be confined in a surface shell for different reasons. For example, if valence neutrons are, in a heavy nucleus, distributed around a high lying principal shell, the corresponding density distribution, for 

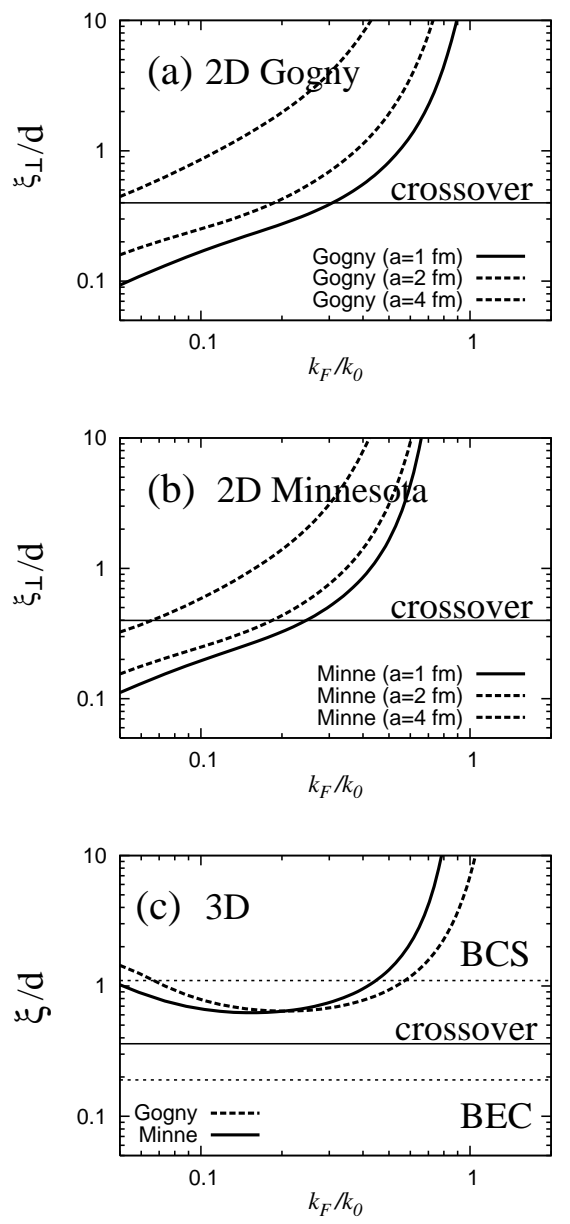

FIG. 7: Ratio of the $n n$-Cooper pair size to the average inter-neutron distance $d$ as a function of $k_{F} / k_{0}\left(k_{0}=1.36\right.$ fm $\left.{ }^{-1}\right)$. (a)(b) Ratio $\xi_{\perp} / d\left(d=\rho^{-1 / 2}\right)$ in quasi-2D neutron matter obtained with the Gogny D1S and Minnesota forces. The $k_{F} \xi_{\perp}=1$ line for a typical value of the BEC-BCS crossover [71, 72, 73] in 2D is shown by solid thin lines. (c) Ratio $\xi / d\left(d=\rho^{-1 / 3}\right)$ in 3D neutron matter with the Gogny D1S and Minnesota forces. The short-dashed lines indicate the boundaries for the weak coupling BCS region $\xi / d>1.1$, the crossover region $0.19 \leq \xi / d \leq 1.1$ and the strong coupling BEC region $\xi / d<0.19$ in the regularized model [27, 74, 75]. Thin solid line is $\xi / d=0.36$ for the unitary limit.

instance if the shell does not contain an $S$ wave, may be concentrated in the surface region. Even though low angular momentum orbits with radial nodes have some strength distribution in the inner region, their probability amplitudes may show peak structure in the surface, and therefore, the pairing is expected to be induced by the surface neutrons due to the possible mixing of many states having significant overlap of their radial part in the surface region.

On the other hand it is also well known that finite size enhances pairing. This is already documented by the fact that the nuclear gap varies on average like $12 / \sqrt{A} \mathrm{MeV}$, that is the presence of a surface certainly plays a role. This finite size enhancement is a quite subtle effect as theoretical investigations show [79] and implies several competing effects. How much the scenario is influenced by quasi 2D features is still an open question but an interesting and suggestive hypothesis which we wanted to explore in this work. So one may consider that the strongly correlated Cooper pairs with about a rms radius of $2 \mathrm{fm}$ live in a surface sheet about $1-2 \mathrm{fm}$ across. In section II we mimicked the transverse motion of the neutrons by a simple frozen Gaussian $\phi_{0}(z)$. The motion perpendicular to $z$ is considered as planar. This is, of course, a highly idealized representation of the situation as mentioned several times. It may nevertheless capture some 2D features which possibly underly the strong pairing correlations in the neutron skin of neutron-rich nuclei.

In the previous analysis of pair size in quasi 2D neutron matter in section IV the BCS-BEC crossover may occur only in dilute 2D matter for densities $\rho \leq 0.05-0.07 \mathrm{fm}^{-2}$. Let us estimate how many small sized Cooper pairs can condensate in an area $4 \pi R^{2}$ of the nuclear surface. For the dineutron condensation, more than one pair should exist at least. From the condition $\rho \leq 0.05-0.07 \mathrm{fm}^{-2}$, two dineutrons can condensate in the surface area for $R \geq 2-3$ 

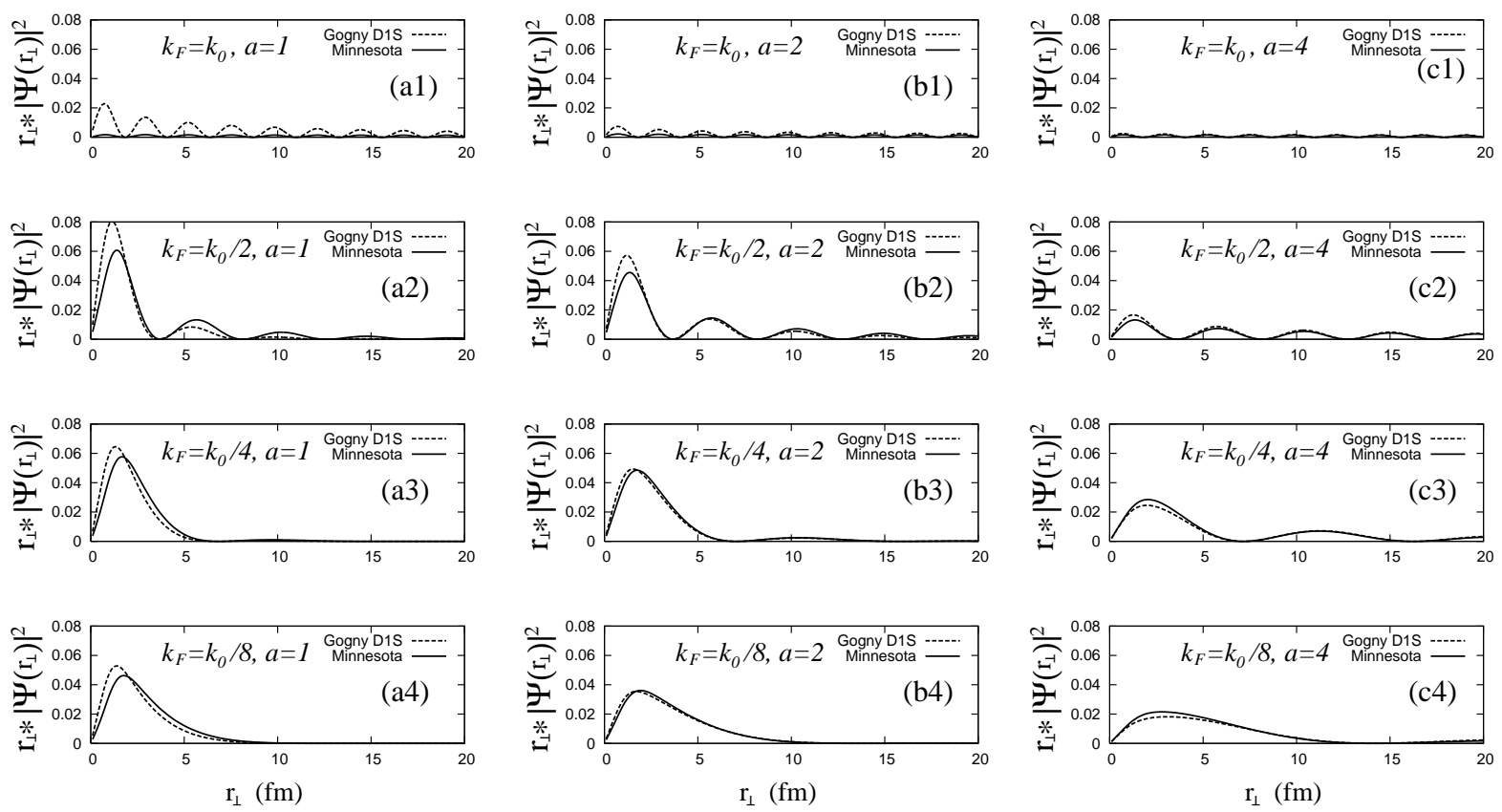

FIG. 8: Probability density $r_{\perp}\left|\Psi_{\text {pair }}\left(r_{\perp}\right)\right|^{2}$ of a $n n$-Cooper pair in the quasi-2D neutron matter as a function of the relative distance $r_{\perp}$. (a1)(a2)(a3)(a4) Probability density in the slab with $a=1 \mathrm{fm}$ at $k_{F}=k_{0}, k_{F}=k_{0} / 2, k_{F}=k_{0} / 4, k_{F}=k_{0} / 8$. (b1)(b2)(b3)(b4) The density in the slab with $a=2 \mathrm{fm} .(\mathrm{c} 1)(\mathrm{c} 2)(\mathrm{c} 3)(\mathrm{c} 4)$ The density in the slab with $a=4 \mathrm{fm}$.

$\mathrm{fm}$, and four dineutrons for $R \geq 3-4 \mathrm{fm}$.

Recently, two-neutron correlations in neutron-skin nuclei have been investigated in light nuclei such as ${ }^{8} \mathrm{He}$ and ${ }^{18} \mathrm{C}[17,18,19]$ as well as medium-heavy nuclei [20, 21]. ${ }^{8} \mathrm{He}$ is considered to be a neutron-skin nucleus having four valence neutrons surrounding an $\alpha$ core, and therefore this nucleus is one of the simple examples to discuss neutron pair correlations in neutron skins. In Refs. 17, 18, 19], it was argued that the ${ }^{8} \mathrm{He}$ ground state has spin-zero twoneutron correlations. From the above-mentioned discussion of quasi-2D neutron matter, the correlation in the ground state is expected to be of BCS-type rather than in the BCS-BEC crossover region, because the valence neutron distribution concentrates in the surface with a distance $R \leq 2 \mathrm{fm}$, which is smaller than the BCS-BEC crossover condition $R \geq 2-3 \mathrm{fm}$ for the condensation of two dineutrons. Instead of the ground state, the possible existence of an excited state ${ }^{8} \mathrm{He}\left(0_{2}^{+}\right)$with a dilute gas-like state of dineutrons was suggested by one of the authors [17]. According to the theoretical results of Ref. [17], the dineutrons exist around the region with $R=4-5 \mathrm{fm}$ which satisfies the above-mentioned condition for the BCS-BEC crossover. Therefore, this state could have BEC-like dineutrons at the surface.

In medium-heavy nuclei, the spatial structure of spin-zero neutron pairing at the surface was investigated based on HFB calculations in Refs. 20, 21]. To discuss two-neutron correlations in the neutron skin in connection with quasi-2D neutron matter, one should analyze the radial and angular behaviour separately and focus on the angular correlations (opening angle) of neutron pairing, as well as on the size of the Cooper pairs. The transition from BCS phase to BCS-BEC crossover is characterized by the change of the pair wave function from a weak-coupling nodal structure to an enhanced single bound state like peak. If such a structure change is found in the angular behaviour it may be an indication of the BCS-BEC crossover, while a clear evidence may not be seen in the radial structure because it might be affected by Pauli effect from constituent neutrons of the core. The spatial structure of spin-zero neutron pairing is displayed in Ref. [20], which shows that the nodal structure along the angular direction is seen inside from the surface and it disappears on the outer side of the surface. More detailed analysis of the angular behaviour is requested. On the other hand, a good measure of the correlations also is the size of the Cooper pairs. Their rms radius seems systematically very small in the outer part of the nuclear surface 21], that is much smaller than the smallest value in homogeneous $3 \mathrm{D}$ matter. Whether this also always goes along with a maximum in the pair correlations at the same place in a finite nucleus, remains to be seen.

Let us also comment on other effects omitted in the present model of infinite quasi-2D neutron matter. At the surface of a realistic finite nuclear system, valence neutrons feel the centrifugal force and the spin-orbit force which may work so as to break $n n$-Cooper pairs. Periodicity condition on the spherical surface is another effect to be 

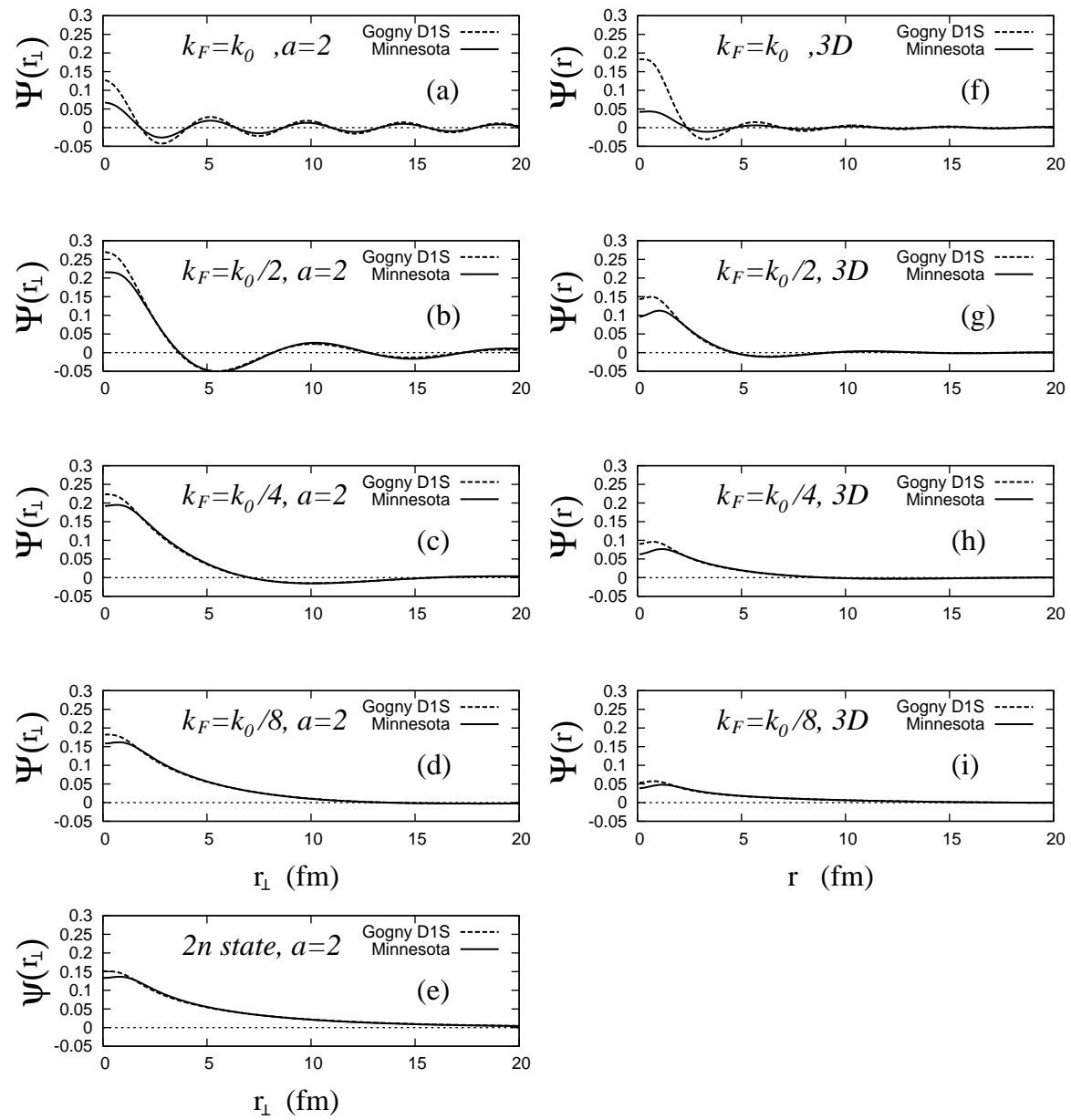

FIG. 9: (a)(b)(c)(d) Pair wave function $\Psi_{\text {pair }}$ of a $n n$-Cooper pair in the quasi-2D neutron matter with $a=2 \mathrm{fm}$ as a function of $r_{\perp}$, and $(\mathrm{f})(\mathrm{g})(\mathrm{h})(\mathrm{i})$ that in 3D neutron matter as a function of $r$. (e) Two-neutron wave function $\psi^{n n}\left(r_{\perp}\right)$ of the bound state in the quasi-2D system with $a=2 \mathrm{fm}$.

considered.

\section{B. Relation to two-neutron halo nuclei}

Two-neutron correlations have extensively been investigated in two-neutron halo nuclei by means of three-body

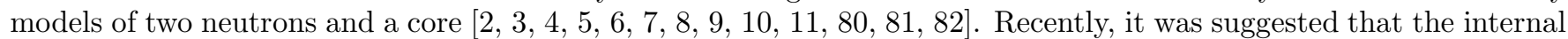
two-neutron wave function analyzed as a function of the distance $R$ from the nuclear center shows behaviour similar to the pair wave function of 3D neutron matter in the BCS-BEC crossover region 77]. In the analysis of Ref. 77], the inter-neutron wave function has a nodal structure at small $R$, and it shows enhancement of the spatial correlations with increasing $R$. Moreover, in a further analysis of the neutron pair in ${ }^{11} \mathrm{Li}$, size shrinking was found at the surface [44]. It was found that there the pair is as small as $3 \mathrm{fm}$ at $R \sim 3 \mathrm{fm}$ [44]. The formation of the small sized dineutron at the surface of ${ }^{11} \mathrm{Li}$ may be understood with the picture of a quasi-2D neutron system. As shown in Ref. [10] with a three-body model, the spin-zero component of two neutrons shows a strong angular correlation and its distribution concentrates at the surface with $R=3 \sim 4 \mathrm{fm}$. The situation corresponds to the quasi-2D neutron where the valence neutrons are confined at the surface region and the neutron pair is formed due to the angular correlation. It may be interpreted as the reduction of the dimension from three to two, being crucial for the small-sized neutron pair in two-neutron halo nuclei. At the small $R$ region, i.e. near the core, the size of the neutron pair increases and nodal structure appears in the relative wave function in the three-body model. This is because the Pauli effect from the constituent neutrons of ${ }^{9} \mathrm{Li}$ core raises the Fermi momentum of the valence neutrons. This behaviour is similar to the weakening of the pair correlation in the BCS region at $k_{F} \geq k_{0} / 2$ in the quasi-2D neutron matter. 
In spite of the analogous behaviour of the neutron pair in ${ }^{11} \mathrm{Li}$ to that of the Cooper pair in the quasi-2D neutron matter, one should keep in mind that two-neutron halo nuclei has only one pair of neutrons and the phenomena there is not regarded as the Cooper pair condensation in the BCS picture. Several investigations have, however, revealed that the features of a single Cooper pair and the ones of a Cooper pair embedded in several others are not drastically different.

\section{Two-proton correlation in quasi-2D systems}

It is also interesting to consider two-proton correlation in proton-rich nuclei in analogy to the two-neutron correlation in neutron-rich nuclei. While the energy of infinite proton matter diverges without charge neutrality, proton-rich matter such as proton halo or proton skin structure can exist in finite nuclei. Moreover, since two-proton decays are allowed from some proton-rich nuclei such as ${ }^{6} \mathrm{Be},{ }^{8} \mathrm{C},{ }^{12} \mathrm{O}$ and ${ }^{16} \mathrm{Ne}[83$, 84, 85], more direct information of $T=1$ pair correlation could be experimentally obtained than for the case of neutron-rich nuclei.

In the same way as neutron systems, we calculate a wave function $\psi^{2 n}\left(r_{\perp}\right)$ for the two-proton bound state in quasi-2D systems. Coulomb force $\left(V_{\text {Coul }}(r)\right)$ is added to the two-body effective nuclear forces. In Fig. 10, the obtained two-proton wave functions in the ${ }^{1} S$-wave channel are shown in comparison with those of the dineutron state. Because of the Coulomb force, the spatial correlation of the $T=1$ pair is slightly more suppressed in the two-proton system than in the two-neutron system. However, the wave functions for $a=1 \mathrm{fm}$ and $a=2 \mathrm{fm}$ still have a pronounced peak. It suggests that the spatial correlation of the $T=1$ pair also is present in proton systems.
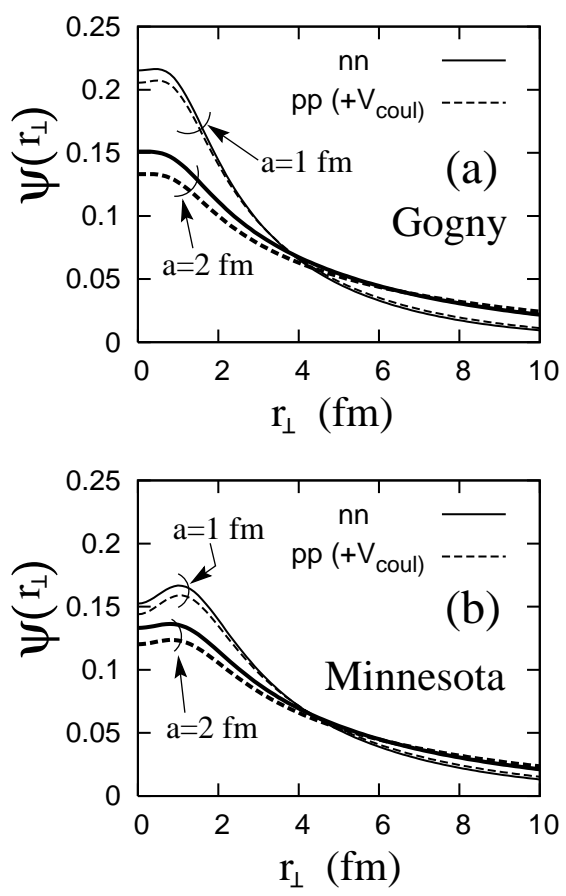

FIG. 10: Two-proton wave functions $\psi^{2 p}\left(r_{\perp}\right)$ in quasi-2D systems with the width parameter $a=1$ and $a=2$ fm calculated with (a) the Gogny force and (b) Minnesota force complemented with Coulomb force ( $\left.V_{\text {Coul }}\right)$. Two-neutron wave functions $\psi^{n n}\left(r_{\perp}\right)$ (without $\left.V_{\text {Coul }}\right)$ are also shown for comparison.

\section{SUMMARY AND OUTLOOK}

Singlet $S\left({ }^{1} S\right) n n$ correlations in quasi-2D neutron systems were studied in a simplified model of neutrons confined in a slab with a certain thickness. The finite-range effective nuclear forces, the Gogny D1S and Minnesota forces, were adopted for the study. The strength of the two-body interaction in the quasi-2D systems depends on the width $a$ of the slab. Namely, the interaction is stronger for thinner slabs than for wider ones. When the width of a slab is small enough, e.g. around $a=1 \mathrm{fm}$, two neutrons form a tightly bound dineutron with a size as small as the deuteron size. 
The behaviour of the $n n$-Cooper pair in the quasi-2D neutron matter was investigated by means of the BCS theory. In quasi-2D neutron matter, the transition region of the BCS-BEC crossover appears at $k_{F} / k_{0}<0.4 \sim 0.5$ and for $a \sim 1 \mathrm{fm}$. The size shrinking of the Cooper pair occurs at finite low $k_{F}$ because of the Pauli effect. It is also important to note that, as the width $a$ of the slab decreases, the pair correlation in $2 \mathrm{D}$ is enhanced and the Cooper pair size becomes small because of the stronger attraction in the thinner slab. This is one of the characteristics different from $3 \mathrm{D}$. In particular, the reduction of the dimension from $3 \mathrm{D}$ to $2 \mathrm{D}$ is crucial to form the strongly correlated neutron pair. The chemical potential becomes negative for $k_{F} / k_{0}<0.2$ and, therefore, there is true two-neutron binding in quasi-2D neutron matter and a genuine BEC regime is born out.

The relation of the quasi-2D neutron matter to finite neutron-rich nuclei was also discussed. Several studies of the past have revealed that the size of Cooper pairs changes very substantially from inside to outside of a nucleus [21, 44]. The subject has been taken up again more recently and the radius dependence of a neutron-neutron $(n n)$ Cooper pair has been investigated more systematically [21, 44]. It thereby turned out that the old believe that the size of a Cooper pair is of the order of the diameter of a large nucleus must be revisited when considered locally, i.e., as a function of the nuclear radius. Namely, detailed BCS calculations with the D1S Gogny force have shown that the rms radius of an $n n$-Cooper pair, though indeed large in the interior, becomes very small in the outer surface of a nucleus, where the density is low, before expanding again when leaving the nucleus. The minimum extension of a Cooper pair attains a size as small as $2 \mathrm{fm}$, that is the same as the size of a deuteron, a bound state. Although the variation of the pair radius as a function of the density qualitatively follows the one of infinite matter, a factor of two reduction of the pair diameter with respect to the homogeneous case is a quite surprisingly strong effect which calls for an explanation.

We considered this pair size shrinking in the surface of neutron-rich nuclei with the present calculations of quasi-2D neutron systems, based on the hypothesis that the valence neutrons in a neutron-rich nucleus live in radial direction in a surface sheet about $1-2 \mathrm{fm}$ across because the wave functions of the active shell are essentially concentrated in the surface while the nucleons are free to move within the sheet. The idea behind this scenario is that the neutrons in the surface sheet are essentially constrained to a two-dimensional world which could give a certain clue to the smallness of the Cooper pairs, since in two dimensions even an isolated $n n$ pair forms a bound state. Pursuing this idea, we further idealized the situation assuming a planar slab of neutrons where for simplicity the motion perpendicular to the slab is frozen into a Gaussian wave packet in order to simulate the surface concentration of the valence neutrons in radial direction. Freezing the perpendicular motion means that the neutrons are two-dimensional in the direction of the slab. The thickness of the slab only influences the strength of the pairing force, as already mentioned above. We, indeed, find that for reasonable slab thicknesses of $a \sim 1 \mathrm{fm}$, the pair radius is of the order of $2-3 \mathrm{fm}$ in agreement with realistic 3D HFB calculations 21]. We are, of course, aware of the fact that our model may be strongly oversimplified and further studies are required in order to ascertain whether a 2D aspect indeed helps to interpret the occurrence of astoundingly small Cooper pairs in the nuclear surface. One also should keep in mind that a local picture may be a little too simple in the sense that the pair size certainly fluctuates a lot. Nevertheless, a classical picture often grasps a good deal of reality, even in so tiny quantum object as nuclei. Neutron pairs much smaller than the dimension of a nucleus may approximately be considered as bosons and a strong coupling Bose-Einstein condensation scenario of $n n$ pairs may be realized in the surface of neutron-rich nuclei. Bound state like Cooper pairs in the nuclear surface may also allow to reinterpret neutron transfer reactions into superfluid nuclei which are known to have a strongly enhanced cross section [86].

$2 \mathrm{D}$ aspects may also be relevant in the Lasagne phase in neutron star crust. In practical application to such systems, one should extend the model by taking into account finite temperature and also excitation in the $z$-direction for higher neutron density.

BCS and BEC problems of two-dimensional fermion systems have been discussed also in the field of condensed matter physics in relation to electron systems and atomic systems [71, 72]. Phenomena of BEC and BCS-BEC crossover in quasi-2D systems of trapped gases have been discussed in recent works [50, 87, 88], and it has been suggested that the condensation in a quasi-2D gas is sensitive to the frequency $\omega$ of the confinement in the "frozen" direction. This feature may have an analogy to the present results for the quasi-2D neutron matter.

\section{Acknowledgements}

The authors would like to thank K. Hagino, H. Horiuchi, N. Pillet, G. Röpke, H. Sagawa and N. Sandulescu for valuable discussions on nuclear pairing and Cooper pairs in the nuclear surface. Fruitful discussions during the YIPQS international molecule workshop held at Yukawa Institute for Theoretical Physics (YITP) in October 2008 were useful to complete this work. The computational calculations of this work were performed by supercomputers at YITP and also by Supercomputer Projects of High Energy Accelerator Research Organization (KEK). This work was supported by Grant-in-Aid for Scientific Research from Japan Society for the Promotion of Science (JSPS). It is also supported 
by the Grant-in-Aid for the Global COE Program "The Next Generation of Physics, Spun from Universality and Emergence" from the Ministry of Education, Culture, Sports, Science and Technology (MEXT) of Japan.

[1] I.Tanihata et al., Phys. Rev. Lett. 55, 2676 (1985).

[2] P. G. Hansen and B. Jonson, Europhys. Lett. 4, 409 (1987).

[3] G. F. Bertsch and H. Esbensen, Annals Phys. 209, 327 (1991).

[4] M. V. Zhukov, B. V. Danilin, D. V. Fedorov, J. M. Bang, I. J. Thompson and J. S. Vaagen, Phys. Rept. 231, 151 (1993).

[5] H. Esbensen, G. F. Bertsch and K. Hencken, Phys. Rev. C 56, 3054 (1997).

[6] K. Ikeda. Nucl. Phys. A538, 355c (1992).

[7] S. Aoyama, K. Katō, and K. Ikeda, Prog. Theor. Phys. Suppl. 142, 35 (2001); T. Myo, S. Aoyama, K. Katō, and K. Ikeda, Prog. Theor. Phys. 108, 133 (2002).

[8] T. Myo, S. Aoyama, K. Katō and K. Ikeda, Prog. Theor. Phys. 108, 133 (2002).

[9] T. Myo, S. Aoyama, K. Katō and K. Ikeda, Phys. Lett. B576, 281 (2003).

[10] K. Hagino and H. Sagawa, Phys. Rev. C 72, 044321 (2005).

[11] T. Myo, K. Katō, H. Toki, K. Ikeda, Phys. Rev. C 76, 024305 (2007).

[12] K. Ieki et al., Phys. Rev. Lett. 70, 730 (1993).

[13] D. Sackett et al., Phys. Rev. C 48, 118 (1993).

[14] S. Shimoura et al., Phys. Lett. B348, 29 (1995).

[15] M. Zinser et al., Nucl. Phys. A619, 151 (1997).

[16] T. Nakamura, et al., Phys. Rev. Lett. 96, 252502 (2006).

[17] Y. Kanada-En'yo, Phys. Rev. C 76, 044323 (2007).

[18] N. Itagaki, M. Ito, K. Arai, S. Aoyama and T. Kokalova, Phys. Rev. C 78, 017306 (2008).

[19] K. Hagino, N. Takahashi and H. Sagawa, Phys. Rev. C 77, 054317 (2008).

[20] M. Matsuo, K. Mizuyama and Y. Serizawa, Phys. Rev. C 71, 064326 (2005).

[21] N. Pillet, N. Sandulescu and P. Schuck, Phys. Rev. C 76, 024310 (2007).

[22] M. Baldo, J. Cugmon, A. Lejeune and U. Lombardo, Nucl. Phys. A515, 409 (1990).

[23] T. Takatsuka and R. Tamagaki, Prog. Theor. Phys. Suppl. 112, 27 (1993).

[24] F. V. De Blasio, M. Hjorth-Jensen, O. Elgaroy, L. Engvik, G. Lazzari, M. Baldo and H. J. Schulze, Phys. Rev. C 56, 2332 (1997).

[25] D. J. Dean and M. Hjorth-Jensen, Rev. Mod. Phys. 75, 607 (2003).

[26] M. Baldo, E. E. Sapershtein and S. V. Tolokonnikov, Nucl. Phys. A749, 42 (2005).

[27] M. Matsuo, Phys. Rev. C 73, 044309 (2006).

[28] J. Margueron, H. Sagawa and K. Hagino, Phys. Rev. C 76, 064316 (2007).

[29] A. A. Isayev, Phys. Rev. C 78, 014306 (2008).

[30] G. F. de Téramond and B. Gabioud, Phys. Rev. C 36, 691 (1987).

[31] A. B. Migdal, Yad. Fiz. 16, 427 (1972); Sov. J. Nucl. Phys. 16, 238 (1973).

[32] H. Bando and A. Kuriyama, Prog. Theor. Phys. Suppl. Extra Number, 69 (1968).

[33] M. Baldo, U. Lombardo and P. Schuck, Phys. Rev. C 52, 975 (1995).

[34] G. Ropke, A. Schnell, P. Schuck and P. Nozieres, Phys. Rev. Lett. 80, 3177 (1998).

[35] U. Lombardo, P. Nozieres, P. Schuck, H. J. Schulze and A. Sedrakian, Phys. Rev. C 64, 064314 (2001).

[36] A. Tohsaki, H. Horiuchi, P. Schuck and G. Ropke, Phys. Rev. Lett. 87, 192501 (2001).

[37] T. Yamada and P. Schuck, Phys. Rev. C 69, 024309 (2004).

[38] Y. Funaki, A. Tohsaki, H. Horiuchi, P. Schuck and G. Ropke, Phys. Rev. C 67, 051306(R) (2003).

[39] P. Ring and P. Schuck, The Nuclear Many-Body Problem (Springer-Verlag, New York, 1980).

[40] D.M. Brink and R.A. Broglia, Nuclear Superfluidity (Cambridge University Press, New York, 2005).

[41] I. Tanihata, D. Hirata, T. Kobayashi, S. Shimoura, K. Sugimoto and H. Toki, Phys. Lett. B289, 261 (1992).

[42] T. Suzuki et al., Phys. Rev. Lett. 75, 3241 (1995).

[43] A. Ozawa, et al., Nucl.Phys. A691, 599(2001).

[44] K. Hagino, H. Sagawa and P. Schuck, arXiv:0812.0658 [nucl-th].

[45] D. G. Ravenhall, C. J. Pethick and J. R. Wilson, Phys. Rev. Lett. 50, 2066 (1983).

[46] C. P. Lorenz, D. G. Ravenhall and C. J. Pethick, Phys. Rev. Lett. 70, 379 (1993).

[47] K. Oyamatsu, Nucl. Phys. A561, 431 (1993).

[48] G. Watanabe, K. Sato, K. Yasuoka and T. Ebisuzaki, Phys. Rev. C 66, 012801(R) (2002); Phys. Rev. C 68, 035806 (2003).

[49] A. I. Safonov, S. A. Vasilyev, I. S. Yasnikov, I. I. Lukashevich and S. Jaakkola, Phys. Rev. Lett. 81, 4545 (1998).

[50] D. S. Petrov, M. Holzmann, and G.V.Shlyapnikov, Phys. Rev. Lett. 84, 2551 (2000).

[51] J. Bardeen, L. N. Cooper and J. R. Schrieffer, J. Bardeen, L. N. Cooper and J. R. Schrieffer, Phys. Rev. 108 1175 (1957).

[52] M. Baldo, U. Lombardo, E. Saperstein and M. Zverev, Phys. Lett. B459, 437 (1999).

[53] M. Farine and P. Schuck, Phys. Lett. B459, 444 (1999).

[54] M. Baldo, M. Farine, U. Lombardo, E. E. Saperstein, P. Schuck and M. V. Zverev, Eur. Phys. J. A 18, 17 (2003).

[55] S. S. Pankratov, M. Baldo, U. Lombardo, E. E. Saperstein and M. V. Zverev, Nucl. Phys. A811, 127 (2008). 
[56] U. Lombardo and P. Schuck, Phys. Rev. C 63, 038201 (2001).

[57] J. Decharge and D. Gogny, Phys. Rev. C 21, 1568 (1980).

[58] J. F. Berger, M. Girod, and D. Gogny, Comput. Phys. Commun. 63, 365 (1991).

[59] H. Kucharek, P. Ring, P. Schuck, R. Bengtsson and M. Girod, Phys. Lett. B216, 249 (1989).

[60] E. Garrido, P. Sarriguren, E. Moya de Guerra and P. Schuck, Phys. Rev. C 60, 064312 (1999).

[61] D. R. Thompson, M. LeMere and Y. C. Tang Nucl. Phys. A286, 53 (1977).

[62] L. D. Landau and E. M. Lifshitz, Quantum Mechanics: Non Relativistic Theory, Course of Theoretical Physics Vol. 3 (Pergamon, New York, 1989).

[63] A. Akmal, V. R. Pandharipande and D. G. Ravenhall, Phys. Rev. C 58, 1804 (1998).

[64] J. Carlson, J. . J. Morales, V. R. Pandharipande and D. G. Ravenhall, Phys. Rev. C 68, 025802 (2003).

[65] H.-J. Schulze, J. Cugnon, A. Lejeune, M. Baldo, and U. Lombardo, Phys. Lett. B375, 1 (1996).

[66] U. Lombardo, P. Schuck and W. Zuo, Phys. Rev. C 64, 021301(R) (2001).

[67] C. Shen, U. Lombardo, P. Schuck, W. Zuo and N. Sandulescu, Phys. Rev. C 67, 061302(R) (2003).

[68] U. Lombardo,P. Schuck and C. Shen, Nucl. Phys. A731, 392 (2004).

[69] L. G. Cao, U. Lombardo and P. Schuck, Phys. Rev. C 74, 064301 (2006).

[70] V. G. J. Stoks, R. A. M. Klomp, C. P. F. Terheggen and J. J. de Swart, Phys. Rev. C 49, 2950 (1994).

[71] M. Randeria, J.-M. Duan, and L.-Y. Shieh, Phys. Rev. Lett. 62, 981 (1989); Phys. Rev. B 41, 327 (1990).

[72] V. M. Loktev, R. N. Quick and S. G. Sharapov, Phys. Rep. 349, 1 (2001).

[73] F. Pistolesi and G. C. Strinati, Phys. Rev. B 53, 15168 (1996).

[74] J. R. Engelbrecht, M. Randeria and C. A. R. Sade Melo, Phys. Rev. B 55, 15153 (1997).

[75] T. Papenbrock and G. F. Bertsch, Phys. Rev. C 59, 2052 (1999).

[76] P. Nozieres and S. Schmitt-Rink, J. Low. Temp. Phys. 59195 (1985).

[77] K. Hagino, H. Sagawa, J. Carbonell and P. Schuck, Phys. Rev. Lett. 99, 022506 (2007).

[78] N. Sandulescu, private communication.

[79] M. Farine, F. W. J. Hekking, P. Schuck and X. Viñas, Phys. Rev. B 68, 024507 (2003).

[80] D. Baye, Nucl. Phys. A627, 305 (1997).

[81] K. Arai, Y. Ogawa, Y. Suzuki and K. Varga, Prog. Theor. Phys. Suppl. No.142, 97 (2001).

[82] P. Descouvemont, E. M. Tursunov and D. Baye, Nucl. Phys. A765, 370 (2006) arXiv:nucl-th/0512016.

[83] L. V. Grigorenko, R. C. Johnson, I. G. Mukha, I. J. Thompson and M. V. Zhukov, Phys. Rev. C 64, 054002 (2001).

[84] F. C. Barker, Phys. Rev. C 68, 054602 (2003).

[85] I. Mukha et al., Phys. Rev. C 77, 061303(R) (2008).

[86] W. von Oertzen, A. Vitturi, Rep. Prog. Phys. 64, 1427 (2001).

[87] L. He and P. Zhuang, Phys. Rev. A 78, 033613 (2008).

[88] W. Zhang. G.-D. Lin and L. -M. Duan, Phys. Rev. A 77, 063613 (2008).

[89] We are aware that the notion 'Cooper pair wave function' has, recently, become a subject of debate (see, e.g., G.G. Dussel, S. Pittel, J. Dukelsky, P. Sarriguren, Phys. Rev. C 76, 011302 (2007) and references in there). We do not want to enter this discussion here and stay with the traditional jargon. 